\title{
Análisis de un caso de inestabilidad lateral de un viga de hormigón pretensado de gran luz en fase de izado
}

\section{Case-study of lateral instability of a long-span prestressed concrete girder during lifting}

\author{
Albert DE LA FUENTE ANTEQUERA* \\ Dr. Ingeniero de Caminos \\ Universidad Politécnica de Cataluña \\ Profesor lector \\ albert.de.la.fuente@upc.edu
}

Sergio Henrique PIALARISSI CAVALARO

Dr. Ingeniero Civil

Universidad Politécnica de Cataluña

Profesor Agregado

sergio.pialarissi@upc.edu

\author{
Jesús Miguel BAIRÁN GARCÍA \\ Dr. Ingeniero de Caminos \\ Universidad Politécnica de Cataluña \\ Profesor Agregado \\ jesus.miguel.bairan@upc.edu
}

* C/Jordi Girona Salgado 1-3, C1-202c, Barcelona (España), CP: 08034. Telf.: 934016515

\section{RESUMEN}

Las mejoras tecnológicas de la prefabricación con hormigón han hecho competitivo el uso de vigas prefabricadas de luces cada vez mayores en comparación con otras alternativas. Sin embargo, el peso de las vigas es limitante en las operaciones de izado y transporte, por lo que se tiende a reducir el ancho de las alas, produciendo elementos más propensos a la inestabilidad lateral. En este artículo se describe un caso de estudio real de inestabilidad lateral de una viga pretensada de puente de gran luz y realizar un estudio paramétrico. Se analizan las actuales provisiones del Código Modelo 2010 y se muestra que pueden resultar inseguros en casos límites.

\section{ABSTRACT}

Improvement in the concrete prefabrication technology makes more competitive the use of longspan concrete girders, compared to other alternatives. However, element's weight is determinant in transportation and lifting operation costs, hence the width the flanges tends to be reduced. This results in elements more prone to lateral instability. In this paper, a real case study of lateral instability of a long precast girder is presented. A parametric analysis is carried out to determine the limits of application of the solution. The current provisions of the Model Code 2010 are analysed and it is shown that they may result insufficient in some limit cases.

PALABRAS CLAVE: Vigas de prefabricadas, Estabilidad lateral, Izado, Falla.

KEYWORDS: Precast girder, Lateral instability, Lifting, Failure

https://doi.org/10.1016/j.hya.2017.04.012 


\section{Introducción}

Las vigas de hormigón prefabricado se emplean con frecuencia en proyectos constructivos condicionados por la rapidez de construcción y complejidad de hormigonado in-situ. Algunos avances tecnológicos (e.g. mejores hormigones, secciones transversales más eficientes) permiten fabricar elementos de longitudes cada vez mayor y los hace más competitivos frente a otras soluciones [1]. Como consecuencia, el peso a manipular en cada elemento es mayor, haciendo que los estados de transporte e izado sean limitantes en términos de diseño estructural.

La estrategia más común para permitir alcanzar luces mayores consiste en minimizar el peso del elemento prefabricado mediante la reducción del ancho de las alas. Sin embargo, ello reduce la rigidez del eje débil y la rigidez a torsión, por lo que aumenta el riesgo de inestabilidad lateral. Tradicionalmente, el problema de inestabilidad lateral en las fases de construcción de elementos de hormigón ha recibido poca atención el proceso de diseño, ya que se consideraba que las secciones son suficientemente compactas y poseen suficiente margen de rigidez lateral. No obstante, recientemente se ha observado un aumento del número y gravedad de los accidentes y daños con vigas prefabricadas esbeltas de hormigón, generando preocupación sobre este problema [2,3].

Los accidentes y daños que se han reportado en la literatura especializada están asociados a excentricidades, en forma de imperfecciones geométricas, que activan el efecto de segundo orden durante ciertas situaciones de carga (izado, transporte y apoyo en elementos elastómeros). El origen de estas excentricidades en vigas prefabricadas incluye [4]: (1) tolerancias de fabricación, (2) variación del posicionamiento lateral del pretensado, (3) fisuración local, (4) fluencia y retracción, (5) soleamiento en una cara lateral, etc. El estudio de la inestablidad laterial de vigas prefabricadas y la determinación de las magnitudes razonables de las imperfecciones [5] se han mostrado de gran importancia para asegurar la seguridad durante las situaciones transitorias de montaje [2].

Los estudios sobre estabilidad de vigas empezaron en la década de 1950 [6-9]. Probablemente, los estudios analíticos y experimentales de Mast [11-12] sean de los más influyentes en el campo. De hecho, el método propouesto por Mast es la base de las comprobaciones actuales del PCI (2016) [13]. Estos estudios fueron los primeros en considerar el efecto de la imperfección lateral inicial y en proporcionar un factor de seguridad para tener en cuenta la reducción de rigidez debido a la fisuración en esta condición. Posteriormente, otros estudios analíticos y numéricos [14-15] proponen un enfoque más general basados en cálculos complejos. Sin embargo, todos estos modelos asumen linealidad de la respuesta del material y desprecian, por lo tanto, la fisuración.

El objetivo de este artículo es avanzar en el estudio del comportamiento de las vigas de hormigón prefabricado soportadas por cables de izado a través de ganchos de izado. Esta situación resulta ser la más desfavorable ya que no existe, en los puntos de apoyo, ninguna restricción al movimiento de sólido rígido de rotación alrededor de la directriz del elemento. De hecho, la estabilidad se alcanza tras una rotación que genera un contrapeso lateral de los extremos en voladizo que compensa el peso del tramo central.

En el artículo se estudia un caso real de fallo por inestabilidad lateral de una viga prefabricada de $45.6 \mathrm{~m}$ de luz. Se utiliza la formulación de Mast $[10,11]$ para justificar los fenómenos observados en la realidad. Se realiza un análisis paramétrico y se estudia la sensibilidad el problema a las 
variables de diseño. Los resultados se cuestionan las previsiones del actual Código Modelo 2010 relativo a los efectos de segundo orden de inestabilidad lateral.

\section{Análisis del proceso de izado}

Los estudios clásicos de inestabilidad lateral asumen la existencia de una restricción al giro lateral en los soportes [10]. El fenómeno se caracteriza por una deformación lateral en la zona central del elemento, produciendo una deformada hacia uno de los lados, lo cual aumenta el momento torsor $\mathrm{y}$, consecuentemente, el giro y la propia deformada lateral. El problema práctico ocurre cuando la deformada no se puede controlar (inestabilidad) o alcanza valores excesivos. Este tipo de inestabilidad es particularmente relevante en vigas metálicas de sección doble-T con baja rigidez a torsión. Las vigas doble-T de hormigón tienen, normalmente, almas más gruesas y alas más anchas, dando lugar a secciones que son entre 100 y 1000 veces más rígidas a torsión que la contrapartida metálica. Por lo tanto, es razonable asumir que las vigas de hormigón son rígidas a torsión. Esto permite simplificar el problema complejo de acoplamiento torsión-flexión, en un problema gobernado por la flexión lateral.

La presencia de imperfecciones laterales $\left(e_{i}\right)$ desplaza ligeramente el centro de gravedad $(G)$ hacia uno de los lados de la directriz de la pieza, el cual actúa como eje de rotación (Figura 1). Consecuentemente, el elemento gira, formando un ángulo $\theta_{\mathrm{i}}$ con el eje vertical. Entonces, una componente del peso (W) se ve aplicado en el eje débil de la sección $(W \sin \theta)$ y produce una deformada lateral, la cual desplaza aún más el centro de gravedad (G). Si existe rigidez suficiente en la dirección débil, se alcanza el equilibrio en una inclinación $\left(\theta_{\mathrm{eq}}\right)$, superior a la inclinación inicial $\left(\theta_{i}\right)$. Por el contrario, si la rigidez lateral no es suficiente, la deformada lateral y la flexión lateral pueden producir la fisuración del elemento o, incluso, la rotura por flexión lateral.

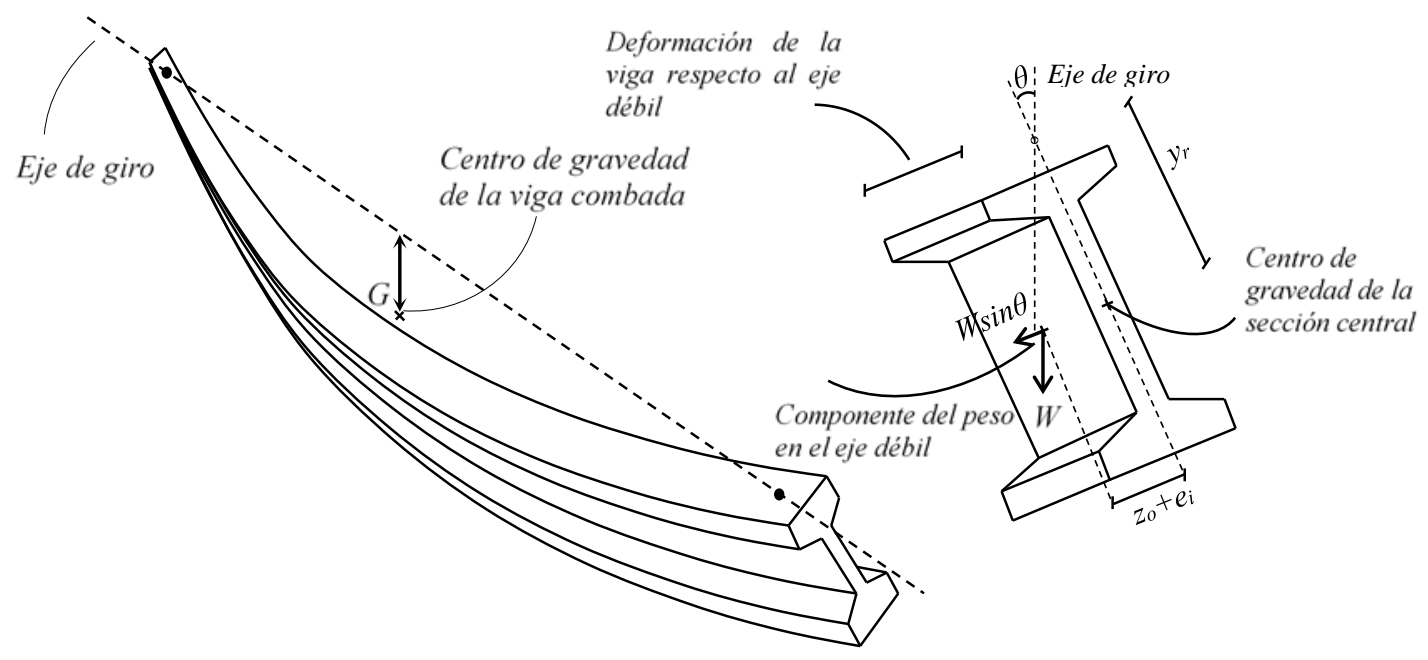

Figura 1. Posición de equilibrio de una viga en situación de izado.

El ángulo de equilibrio $\left(\theta_{\mathrm{eq}}\right)$ puede ser evaluado mediante iteraciones sucesivas usando la ecuación

1. Donde, $y_{r}$ es la atura del punto de eje de giro sobre el centro de gravedad de la sección (G), ver Figura 1, $Z_{0}$ es la deformada lateral, evaluada a la altura de $G$, debido al peso completo de la viga (W) aplicado en el eje débil.

$$
\tan \theta_{e q}=\frac{z_{o} \sin \theta_{e q}+e_{i}}{y_{r}}
$$


Los efectos negativos de estabilidad que puede producir la contraflecha de pretensado, se pueden tener en cuenta desplazando hacia arriba el centro de gravedad $(G)$ una distancia $\delta_{G}=2 / 3 \delta_{P}$. Donde $\delta_{\mathrm{P}}$ es la contraflecha en el centro luz que se espera durante las operaciones de izado). Por otro lado, se puede calcular $z_{0}$ mediante la ecuación 2. En ella $L$ es la luz total del elemento, a es la distancia de los ganchos de izado, desde los extremos de la viga, y $L_{1}$ es la luz central $\left(L_{1}=L-2 a\right)$. Así mismo, E es el módulo de deformación del hormigón e $\mathrm{I}_{y y}$ es la inercia en el eje débil.

$$
\mathrm{z}_{\mathrm{o}}=\frac{\mathrm{W}}{12 \mathrm{EI}_{\mathrm{yy}} \mathrm{L}}\left(\frac{1}{10} \mathrm{~L}_{1}^{5}-\mathrm{a}^{2} \mathrm{~L}_{1}^{3}+3 \mathrm{a}^{2} \mathrm{~L}_{1}+\frac{6}{5} \mathrm{a}^{5}\right)
$$

El margen de seguridad frente a la fisuración $\left(\mathrm{SF}_{\mathrm{cr}}\right)$ viene dado por la ecuación 3. Donde, bajo la hipótesis de viga rígida a torsión, $\theta_{\mathrm{i}}=\mathrm{e}_{\mathrm{i}} / \mathrm{y}_{\mathrm{r}}$ y $\theta_{\mathrm{cr}}$ es el ángulo de rotación de la sección de centro luz en el que se espera que ocurra la fisuración. $\theta_{\text {cr }}$ se puede calcular como el ángulo en el que es necesario rotar la sección hasta que aparece una tensión de tracción en el hormigón igual a la resistencia media de tracción $\left(\mathrm{f}_{\mathrm{ctm}}\right)$. La subrutina de análisis seccional presentada en [16] ha sido usada para calcular $\theta_{\text {cr. }}$ Nótese que $\mathrm{SF}_{\mathrm{cr}}$ combina, simultáneamente, los efectos de estabilidad lateral $\left(\mathrm{z}_{\mathrm{o}} / \mathrm{y}_{\mathrm{r}}\right)$ y los efectos de la flexión lateral en la fisuración $\left(\theta_{i} / \theta_{\mathrm{cr}}\right)$.

$$
\mathrm{SF}_{\mathrm{cr}}=\frac{1}{\frac{\mathrm{z}_{\mathrm{o}}}{\mathrm{y}_{\mathrm{r}}}+\frac{\theta_{\mathrm{i}}}{\theta_{\mathrm{cr}}}}
$$

\section{Caso de estudio}

\subsection{Introducción}

El caso consiste en una viga de puente de $45.6 \mathrm{~m}$ de longitud, prefabricada, de hormigón pretensado, de sección doble T y geometría prismática (ver Figura 2). El área de la sección ( $\left.A_{c}\right)$ es $0.58 \mathrm{~m}^{2}$, la inercia en los ejes fuerte $\left(\mathrm{I}_{\mathrm{xx}}\right)$ y débil $\left(\mathrm{I}_{\mathrm{yy}}\right)$ son $0.325 \mathrm{~m}^{4}$ y $0.021 \mathrm{~m}^{4}$, respectivamente. La viga fue izada usando ganchos dobles, localizados a $2.0 \mathrm{~m}$ desde los extremos (a) y a una altura de $0.30 \mathrm{~m}$ sobre el borde superior de la pieza (Figura 3). Se usaron dos grúas durante las operaciones de izado para garantizar el alineamiento vertical de los cables de izado $\left(\Psi=0^{\circ}\right)$.
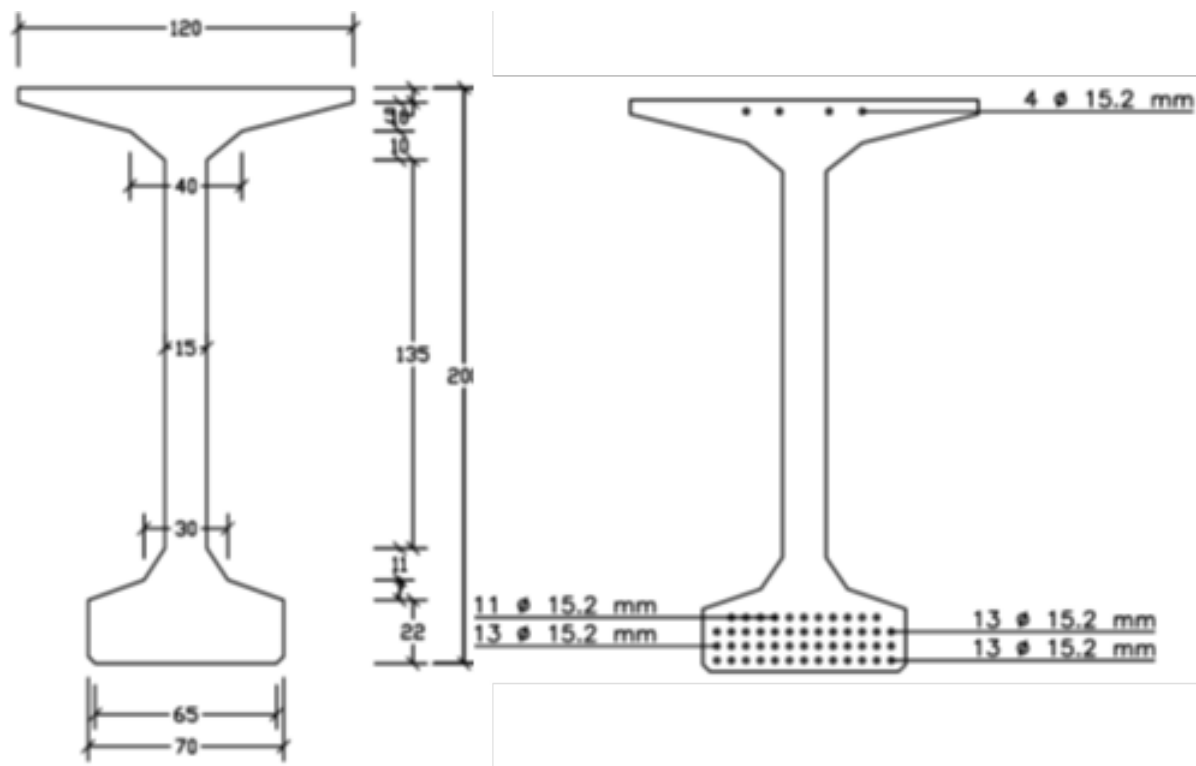

Figura 2. Dimensiones (cm) y armadura activa de la sección transversal. 


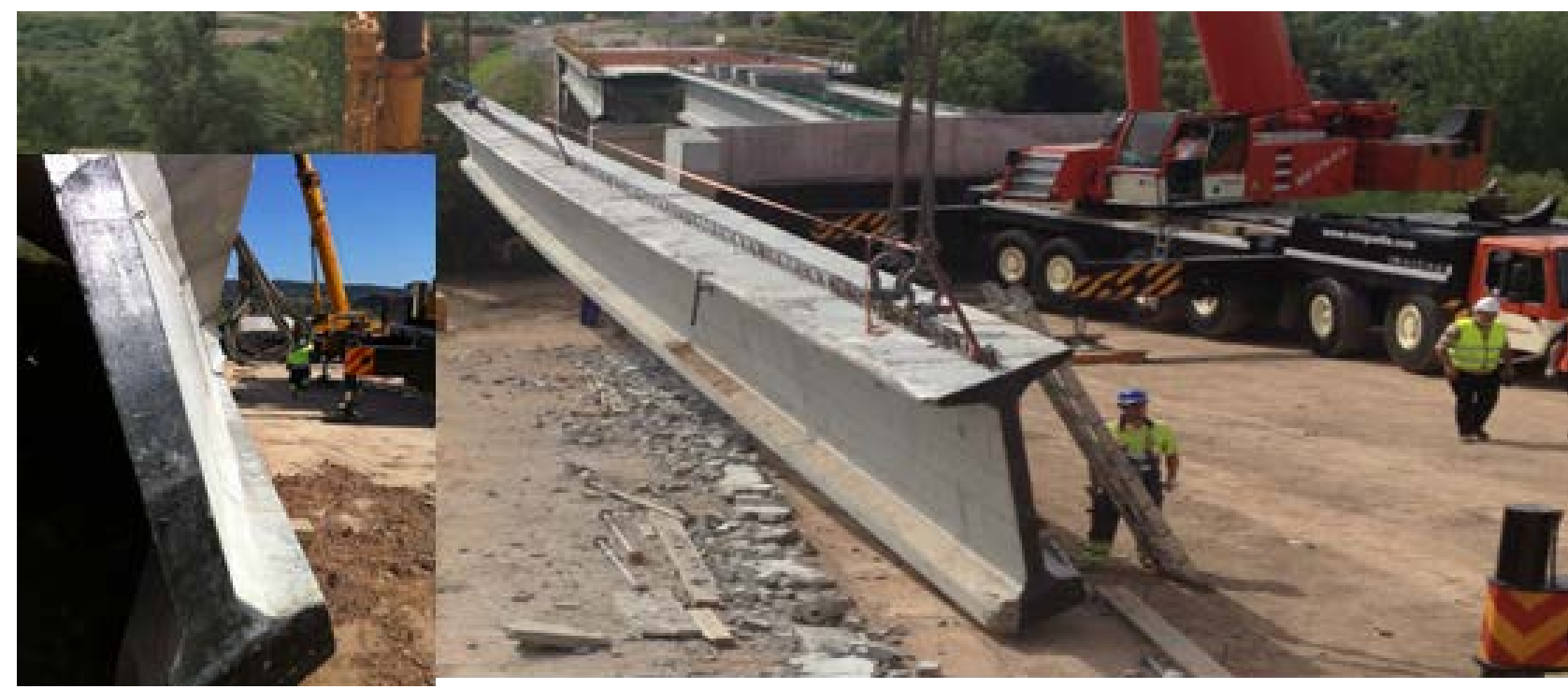

Figura 3. Configuración de izado y deformada.

La fuerza de pretensado en el momento de izado ( $\mathrm{t}>28$ días) se estimó en $\mathrm{P}_{\mathrm{k}}=8514 \mathrm{kN}$ (15\% pérdidas). La excentricidad respecto al centro de gravedad de la sección es $e_{p}=0.73 \mathrm{~m}$. La resistencia del hormigón a los 28 días es de $60 \mathrm{MPa}$. El módulo de deformación y la resistencia media a tracción se estimaron como, $\mathrm{E}_{\mathrm{c}}=34.7 \mathrm{GPa}$ y $\mathrm{f}_{\mathrm{ctm}}=4.53 \mathrm{MPa}$, respectivamente.

En las inspecciones visuales previas al izado, se detectó una excentricidad de, aproximadamente, $12 \mathrm{~mm}$ en la posición de los ganchos de izado respecto al eje central de la pieza. Así mismo, se observó una imperfección en la flecha lateral de $90 \mathrm{~mm}$. Esta imperfección equivale a L/510, la cual es menor que la tolerancia permitida (L/500) en EN 15050 [17]; por lo tanto, se autorizó el izado de la pieza. Durante la operación, se observó un desplome (Figura 3) con un dramático incremento del desplazamiento lateral de hasta $300 \mathrm{~mm}$, por lo que se bajó la viga y se abortó la operación.

En las inspecciones posteriores, se detectaron fisuras verticales en el ala superior izquierda, donde se esperaban las máximas tensiones de tracción debidas al fenómeno de vuelco lateral. A pesar de que los anchos de fisura observados eran aceptables, la viga no recupero su forma original, y la flecha lateral permanente superaba $L / 400$, por lo que el elemento fue finalmente rechazado.

\subsection{Análisis con un modelo de Elementos Finitos}

Se llevó a cabo un análisis numérico del proceso de izado de la viga descrita mediante el software DIANA. El modelo estaba compuesto por elementos solidos isoparamétricos piramidales de 4 nodos, con un tamaño máximo de $230 \mathrm{~mm}$. Los materiales y sus propiedades se simularon teniendo en cuenta los parámetros definidos en el apartado 3.1. La no lineal geométrica se tuvo en cuenta mediante el método de Lagrange.

En la Figura 4a se presenta la configuración deformada de la viga en fase de izado. La deformación lateral alcanzada por el modelo es de $310 \mathrm{~mm}$, ligeramente superior a los $300 \mathrm{~mm}$ observados en las operaciones reales de izado de la viga. Por tanto, se confirma la tendencia a las grandes deformaciones laterales y a la fisuración de la misma en esta fase transitoria (tensiones superiores $a \mathrm{f}_{\mathrm{ctm}}=4.5 \mathrm{MPa}$ en el ala superior y parte externa del ala inferior), según la distribución de tensiones en la sección central de la viga (Figura 4b). 
a)

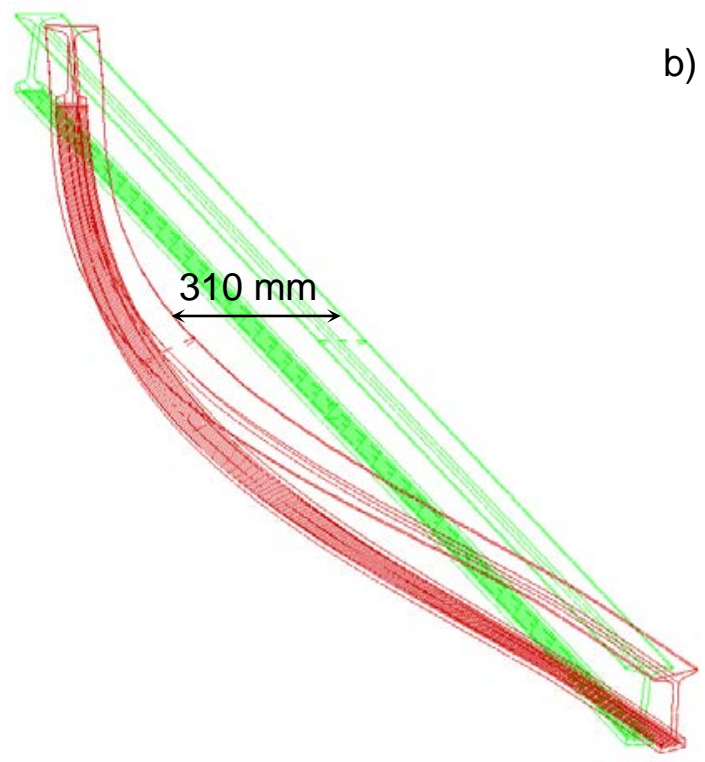

b)

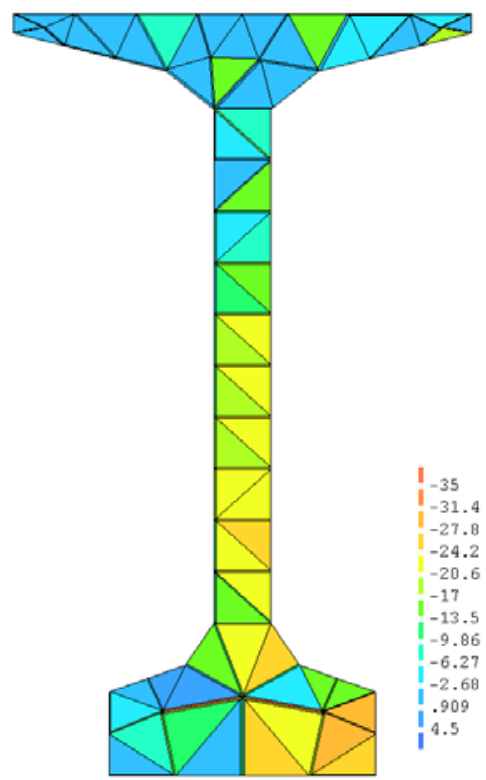

Figura 4. Resultados del modelo numérico: (a) configuración deformada y (b) estado tensional de la sección central (en MPa).

\subsection{Análisis con el modelo de Mast}

Se realizó un estudio paramétrico considerando el rango de valores de las imperfecciones, usando la formulación de Mast $[10,11]$, tal y como se describió en el apartado anterior. Se consideraron deformaciones iniciales entre L/250 (182 mm) y L/1000 (45.6 mm). La excentricidad total del centro de gravedad de la viga $\left(e_{i}\right)$ se consideraron iguales a la flecha lateral, añadiendo una excentricidad lateral de los ganchos de izado $(12 \mathrm{~mm})$.

En la Tabla 1 se presentan los resultados en términos del ángulo de equilibrio en la sección central $\left(\theta_{\text {eq }}\right)$, el ángulo que produce la fisuración $\left(\theta_{\mathrm{cr}}\right)$, el ratio tensión máxima de tracción frente a la resistencia media de tracción $\left(\sigma_{\mathrm{ct}, \mathrm{max}} / \mathrm{f}_{\mathrm{ctm}}\right)$ y el margen de seguridad frente a la fisuración $\left(\mathrm{SF}_{\mathrm{cr}}\right)$.

Los resultados de la Tabla 1 confirman que la imperfección inicial L/500 (91 mm) es suficiente para producir un ángulo de equilibrio $\theta_{\mathrm{eq}}=5.36^{\circ}$ y $\mathrm{SF}_{\mathrm{cr}}=1.07$, el cual satisface las recomendaciones del $\mathrm{PCl}$ (2016) ( $\left.\mathrm{SF}_{\mathrm{cr}} \geq 1.00\right)$. No obstante, se deduce que la viga es susceptible de fisurar ya que margen de seguridad está muy cerca del límite.

\begin{tabular}{rcccc}
\cline { 2 - 5 } & $\mathrm{L} / 250$ & $\mathrm{~L} / 500$ & $\mathrm{~L} / 750$ & $\mathrm{~L} / 1000$ \\
\hline $\boldsymbol{\theta}_{\text {eq }}$ & $14.87^{\circ}$ & $5.36^{\circ}$ & $3.88^{\circ}$ & $3.13^{\circ}$ \\
$\boldsymbol{\sigma}_{c t, \text { max }} \mid \boldsymbol{f}_{c t m}$ & 4.14 & 0.80 & 0.27 & 0.01 \\
$\boldsymbol{\theta}_{c r}$ & $5.94^{\circ}$ & $5.94^{\circ}$ & $5.94^{\circ}$ & $5.94^{\circ}$ \\
$\mathbf{S F}_{\mathrm{cr}}$ & 0.61 & 1.07 & 1.28 & 1.43
\end{tabular}

Tabla 1. Resultados del estudio de sensibilidad

Para profundizar en el análisis, la Figura 5 muestra la evolución de $\theta_{\text {eq }}$ y la combadura (L/e $\mathrm{e}_{\mathrm{i}}$. En la Figura 4 se evidencia que $\theta_{\text {eq }}$ es fuertemente dependiente en $L / e_{i}$. Se observan valores de $\theta_{\text {eq }}$ entre $3.13^{\circ}(\mathrm{L} / 1000)$ a $14.87^{\circ}(\mathrm{L} / 250)$. Se debe hacer notar que $\theta_{\text {eq }}$ aumenta exponencialmente para 
L/e $<600$. Así mismo, $\theta_{\text {cr }}$ es casi $10 \%$ mayor que el valor esperado para $L / 500\left(\theta_{\text {eq }}=5.36^{\circ}\right)$. De hecho, se espera que la viga fisura para una imperfección lateral de L/475. Este valor se puede alcanzar fácilmente previamente a las operaciones de izado, debido a fenómenos como gradientes térmicos (radiación solar) acoplados con las deformaciones de retracción y fluencia.

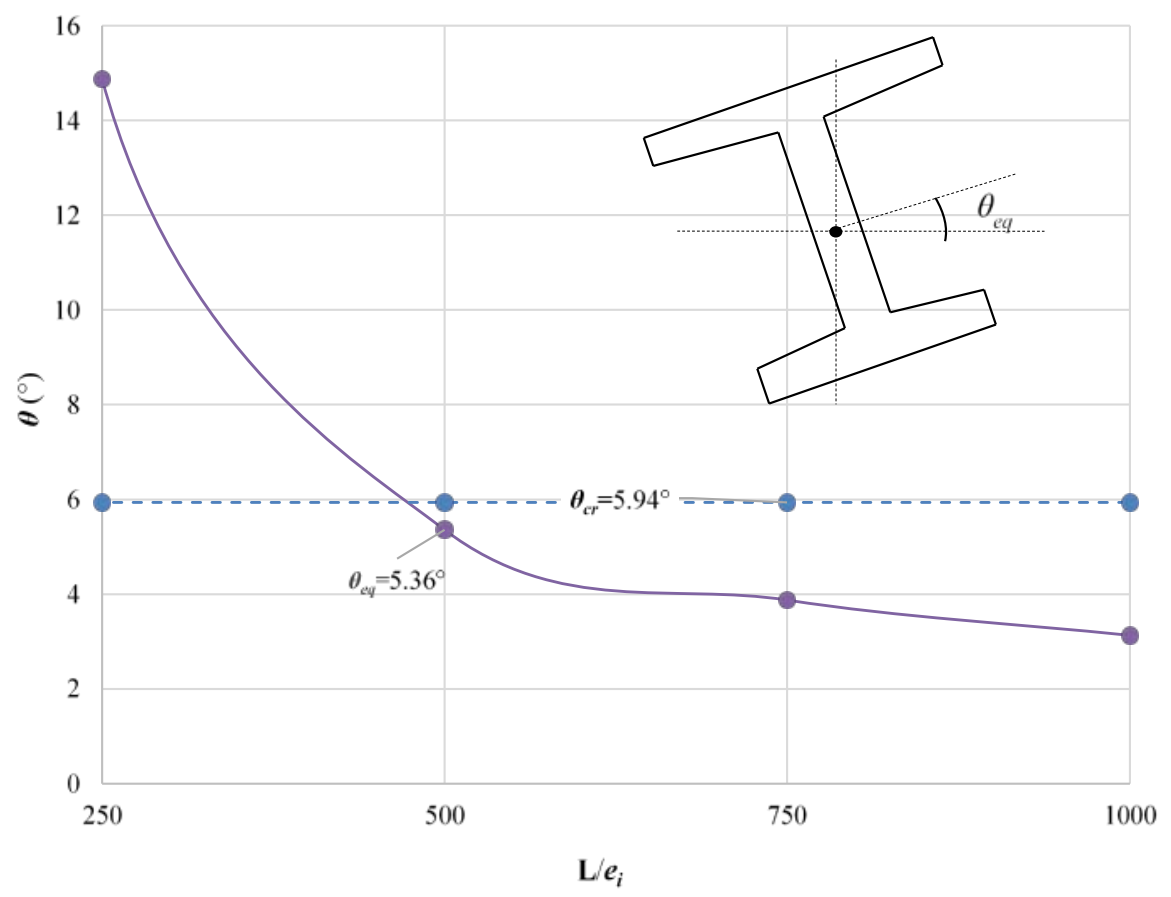

Figura 5. Relación entre $\theta_{\text {eq }}$ y $L / e_{i}$.

La Figura 6 muestra la relación entre el ratio de tensión máxima de tracción y la resistencia media de tracción y el margen de seguridad $\mathrm{SF}_{\mathrm{cr}}$ en función de $\mathrm{L} / \mathrm{e}_{\mathrm{i}}$. Se puede observar que $\sigma_{\mathrm{ct} \text {,max }} / \mathrm{f}_{\mathrm{ctm}}$ aumenta exponencialmente con valores $\mathrm{L} / \mathrm{e}_{\mathrm{i}}<500$. En efecto, para $\mathrm{L} / 500, \sigma_{\mathrm{ct} \text {,max }}$ está $20 \%$ por encima de $f_{c t m}$ y $S_{c r}$ es de 1.07. Por tanto, el riesgo de fisuración en el ala superior existe. Los resultados y análisis confirman que la configuración de izado hace la viga susceptible a la fisuración y a la inestabilidad lateral.

\subsection{Comparación con normativas}

EI Anejo 11 de la Instrucción española EHE-08 [18] establece una tolerancia a la imperfección lateral de L/750, el cual conduce a $S_{\mathrm{cr}}>1.25$. Por el contrario, el Código Modelo (MC-2010) [19] sugiere, en su sección 7.3.8, que se considera una imperfección lateral de L/300 en el análisis de estabilidad, lo cual no sería aceptable para la viga estudiada, de acuerdo a los resultados presentados. El MC2010 también sugiere que los efectos de segundo orden pueden ser despreciados si se cumple L/b $\leq 50 /(\mathrm{h} / \mathrm{b})^{1 / 3}$; siendo b el ancho del ala superior y $\mathrm{h}$ la altura de la sección, respectivamente. El caso de estudio aquí presentado cumple esta última condición con $\mathrm{L} / \mathrm{b}=38<50 /(\mathrm{h} / \mathrm{b})^{1 / 3}=42$. Así pues, se resalta que el cumplimiento de los requisitos en los códigos actuales no garantiza la inexistencia de problemas de inestabilidad lateral.

\section{Conclusiones}

Este estudio evidencia que la tendencia actual de usar vigas prefabricadas de hormigón pretensado para luces cada vez mayores, manteniendo la misma sección transversal de los catálogos 
existentes, aumenta el riesgo de inestabilidad lateral. Es necesario tomar medidas adicionales para evitar este problema.

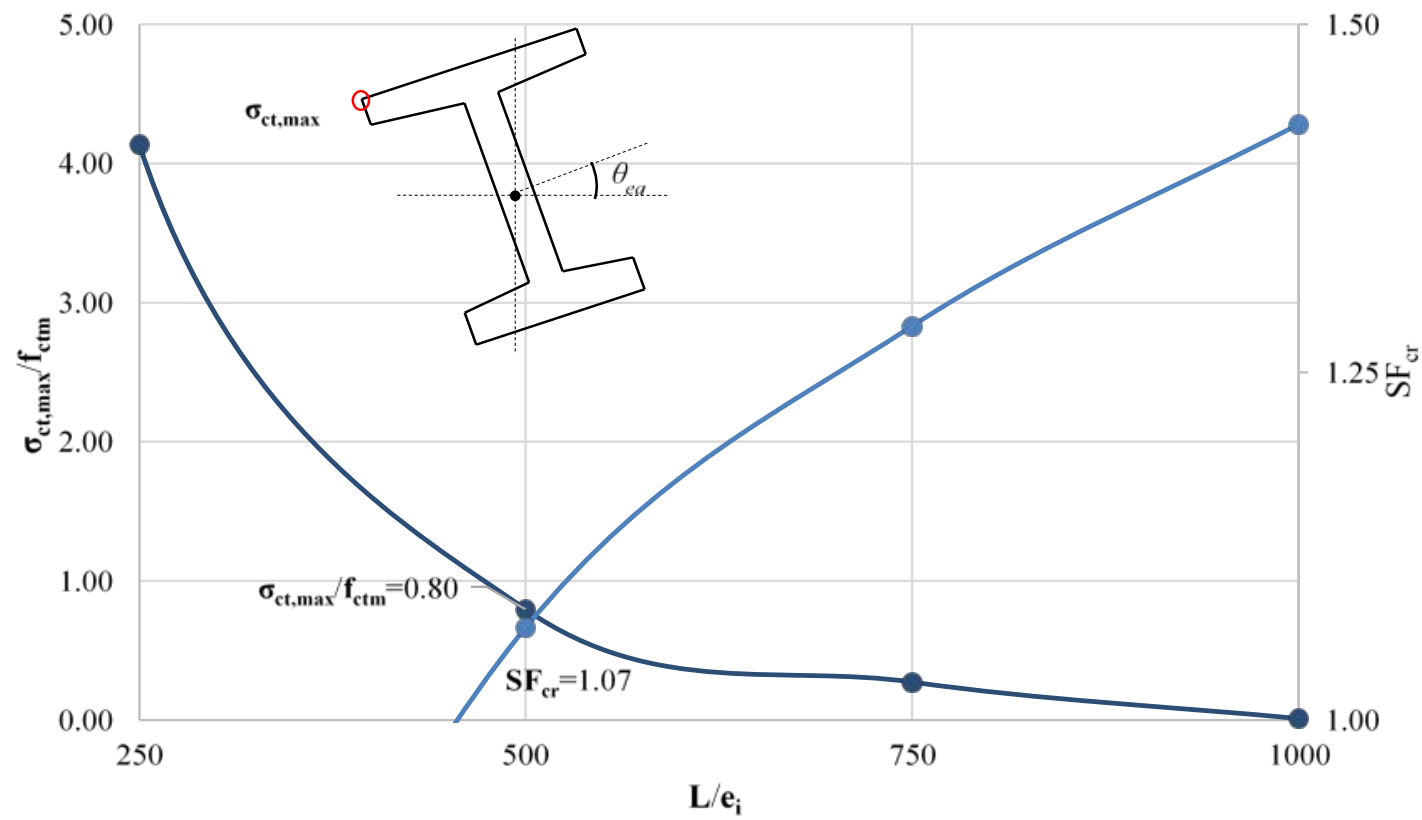

Figura 6. Relación entre $\sigma_{c t, \max } / f_{c t m}-L / e_{i}$ y $S F_{c r}-L / e_{i}$.

A pesar de cumplir los requisitos de imperfecciones geométricas de las normativas EN-15050 y MC2010, el caso de estudio analizado mostró problemas de fisuración y no recobró la deformación inicial después de haber sufrido problemas de inestabilidad lateral durante el izado. El análisis llevado a cabo con las hipótesis descritas en este artículo confirman que existe un margen de seguridad muy pequeño durante el proceso de izado.

Los resultados sugieren que las tolerancias geométricas permitidas y los criterios para determinar la influencia de los efectos de segundo orden que existen en las normativas vigentes deberían ser revisados para tener en cuenta las tendencias actuales en la esbeltez lateral de los elementos. En este sentido, cabe resaltar que el TG 6.5 Puentes Prefabricados de la fib está actualmente proponiendo nuevos requerimientos adaptados a las prácticas actuales de prefabricación con hormigón.

\section{Agradecimientos}

Los autores de esta comunicación quieren agradecer a Don David Fernández-Ordóñez, Dr. Ingeniero de Caminos y actual secretario general de la fib, por las sugerencias aportadas para el desarrollo de este trabajo.

\section{Referencias}

[1] Castrodale, R. W. and White, C. D. "Extending Span Ranges of Precast Prestressed Concrete Girders". National Cooperative Highway Research Program, Report 517. Washington D. C., 2004.

[2] Hurff, J. "Stability of Precast Prestressed Concrete Bridge Girders Considering Imperfections and 
Thermal Effects". Georgia Institute of Technology, School of Civil and Environmental Engineering, August 2010.

[3] Rose, J. "Marquam Bridge wreck likely caused by truck with massive beam braking for Portland traffic jam". The Oregonian, April 2013.

[4] Bairán, J. M. and Cladera, A. (2014). "Collapse of a precast concrete beam for a light roof. Importance of elastomeric bearing pads in the element's stability." Eng. Failure Analysis, 39, 188-199.

[5] PCI. Tolerance Manual for Precast and Prestressed Concrete Construction. Precast Concrete Institute, Chicago, IL. $1^{\text {st }}$ ed., 2000.

[6] Muller, J. "Lateral Stability of Precast Members During Handling and Placing". PCI Journal, February 1962, pp. 20-31.

[7] Anderson, A. R. "Lateral stability of long prestressed concrete beams". PCl Journal, 16(3), 7-9. May-June 1971.

[8] Swann, R. A., Readers comment to "Lateral Stability of Long Prestressed Concrete Beams", PCl Journal, 16(6), November-December 1971.

[9] Laszlo, G. and Imper, R. R. "Handling and Shipping of Long Span Bridge Beams". PCI Journal, November-December 1987, pp. 86-101.

[10] Mast, R. F. (1989). "Lateral Stability of Long Prestressed Concrete Beams, Part 1." PCI J. 34(1), 34-53.

[11] Mast, R. F. (1993). "Lateral Stability of Long Prestressed Concrete Beams, Part 2." PCI J., 38(1), 70-88.

[12] Mast, R. F. (1994). "Lateral bending test to destruction of a $149 \mathrm{ft}$. prestressed concrete I-beam." PCl J., 39(1), 54-62.

[13] PCI. Recommended practice for lateral stability of precast, prestressed concrete bridge girders (CB-02-16). PCl committee on Bridges. 2016.

[14] Stratford, T. J., and Burgoyne, C. J. (2000). "The toppling of hanging beams." Int. J. Solids Struct., 27(26), 3569-3589.

[15] Plaut, R. H., and Moen, C. D. (2013). "Analysis of elastic, doubly symmetric, horizontally curved beams during lifting." J. Struct. Eng., 139(1), 39-46.

[16] de la Fuente, A., Aguado, A., Molins, C., and Armengou, J. (2012). "Numerical model for the analysis up to failure of precast concrete sections." Comput. Struct.,106-107, 105-14.

[17] EN 15050:2008 +A1:2012. Precast concrete products - Bridge elements.

[18] EHE-08. Comisión Permanente del Hormigón. Instrucción de Hormigón Estructural. Ministerio de Fomento. $3^{\text {th }}$ Edition, 2009.

[19] fib Bulletins 65-66, Model Code 2010. Final Draft, 2010. fédération internationale du béton (fib), Lausanne, Switzerland. 